\title{
АНАЛИЗ ВЫПОЛНЕНИЯ СМЕТЫ КАК СПОСОБ ЭФФЕКТИВНОГО КОНТРОЛЯ ИСПОЛЬЗОВАНИЯ БЮДЖЕТНЫХ СРЕДСТВ АВТОНОМНОГО УЧРЕЖДЕНИЯ
}

\section{ANALYSIS OF THE ESTIMATE EXECUTION AS A METHOD OF EFFECTIVE CONTROL OF THE USE OF BUDGETARY FUNDS OF AN AUTONOMOUS INSTITUTION}

\section{T. Turishcheva}

Summary: The article is devoted to the study of topical issues related to the features of the analysis of the implementation of the estimate as a way to effectively control the use of budgetary funds of an autonomous institution. In the course of the research, a separate emphasis is placed on the specifics of the financial support of autonomous institutions, which implies the content and types of analytical procedures. In addition, the stages of analysis of the implementation of the budget of an autonomous institution are highlighted. Particular attention is paid to the techniques and methods of assessing the effectiveness of the use of budgetary allocations allocated to autonomous institutions.

Keywords: autonomous institution, appropriations, estimate, analysis, efficiency, comparison.

\author{
Турищева Татьяна Борисовна \\ к.э.н., дочент, РЭА им. Г.В. Плеханова; Финансовый \\ Университет при Правительстве Рф \\ ttb2812@mail.ru
}

Аннотация: Статья посвящена изучению актуальных вопросов, связанных с особенностями проведения анализа выполнения сметы как способа эффективного контроля использования бюджетных средств автономного учреждения. В процессе исследования отдельный акцент сделан на специфике финансового обеспечения автономных учреждений, которая предопределяет наполнение и виды аналитических процедур. Кроме того, выделены этапы анализа выполнения сметы автономного учреждения. Особое внимание уделено приемам и способам оценки эффективности использования бюджетных ассигнований, выделяемых автономным учреждениям.

Ключевые слова: автономное учреждение, ассигнования, смета, анализ, эффективность, сравнение.
$\mathrm{B}$ современных условиях рационализации распределения бюджетных средств, необходимости постоянного поиска резервов повышения эффективности их использования, возникает необходимость обоснованного и целевого расходования имеющихся ресурсов, необходимых для осуществления эффективной деятельности предприятий и организаций, финансируемых из государственного бюджета, к числу которых относятся автономные учреждения [1].

Одной из важнейших функций управления в бюджетном процессе наряду с планированием, организацией, учетом и контролем является анализ. С учетом вышеизложенного, особую актуальность приобретает проблема научных исследований методики экономического анализа выполнения сметы автономных учреждений и разработка способов внедрения такой методики в практическую деятельность.

При этом, необходимо отметить, что расходы автономных учреждений, которые они несут в процессе оказания услуг, выполнения работ по своему экономическому наполнению существенно отличаются от расходов других предприятий и являются одним из главных и важнейших показателей деятельности данных учреждений.
Анализ сметы дает возможность оценить эффективность использования выделенных учреждению ресурсов, выяснить причины недостачи или же перерасход бюджетных средств, а, следовательно, осуществлять контроль за рациональным их расходованием с целью эффективного управления автономным учреждением.

Отдавая должное научным достижениям ученых, следует отметить, что проблема разработки новых подходов к анализу выполнения сметы автономных учреждений в условиях углубления рыночных трансформаций и ограниченности бюджетных средств требует дальнейшей научно-практической проработки. В значительной степени это обусловлено трансформационными процессами, направленными на достижение международных стандартов осуществления экономической деятельности субъектами хозяйствования.

Таким образом, указанные обстоятельства предопределяют выбор темы данной статьи.

Значительный вклад в теорию, методологию и практику учета и анализа в бюджетных учреждениях внесли такие ученые как: Атамас П.И., Болюх М.А., Вербило А.Ф., Джога Р.Т., Деркач Н.И., Жук В.Н., Калюга Е.В., Опарина 


\section{В.Н., Сушко Н.И. и др.}

Проблемам анализа бюджетных учреждений с разных точек зрения в своих трудах уделяли внимание следующие специалисты: Гончарова Н.Н., Аукина А.И., Федотова Е.С., Макарова Е.Н., Осипова К.И.

В тоже время, несмотря на значительный вклад ученых в развитие теории и практики учета и анализа в бюджетных учреждений в целом и автономных учреждениях в частности, необходимо отметить, что часть имеющихся публикаций является уже устаревшей, к тому же исследователи зачастую используют разрозненные подходы к методике анализа. Также представляются необходимыми дальнейшие научные исследования, посвященные разработке аналитических подходов, которые будут учитывать различную отраслевую специфику деятельности автономных учреждений.

C учетом вышеизложенного, цель статьи можно сформулировать следующим образом: рассмотреть особенности, инструменты и методы проведения анализа выполнения сметы как способа эффективного контроля за использованием бюджетных средств автономного учреждения.

Согласно Закону РФ «Об автономных учреждениях», автономное учреждение является некоммерческой организацией, которая создается государством на федеральном, региональном уровне, также может создаваться на уровне муниципалитетов с целью выполнения работ и оказания услуг в социальной сфере в пределах определенных законодательством полномочий государственной и муниципальной власти.

Основным плановым документом автономных учреждений, который подтверждает их полномочия по получения доходов и осуществлению расходов, а также определяет объем и направление расходования средств для выполнения ими своих функций и достижения целей, определенных на год в соответствии с бюджетными назначениями, является смета [2]. Разработка сметы автономного учреждения направлена на решение двух основных задач: во-первых, определение объема доходов и расходов, связанных с его деятельностью или деятельностью отдельных структурных единиц и подразделений, а во-вторых, обеспечение покрытия расходов финансовыми ресурсами из разных источников.

Ключевым аспектом анализа выполнения сметы как способа эффективного контроля использования бюджетных средств автономного учреждения, по мнению автора, является учет особенностей их финансирования, которое осуществляется за счет бюджетных ассигнований и внебюджетных поступлений.
Финансирование деятельности, осуществляемой автономными учреждениями в соответствии с государственным (муниципальным) заданием и (или) обязательствами перед страховщиком по обязательному социальному страхованию, осуществляется в виде субсидий из соответствующего бюджета бюджетной системы Российской Федерации и иных не запрещенных федеральными законами источников. Автономные учреждения также имеют право зарабатывать самостоятельно, выполняя работы, оказывая услуги, относящиеся к его деятельности, для граждан и юридических лиц за плату. (ст.4 Закона № 174-Ф3).

Размер этих фондов строго планируется и расходуется в соответствии с утвержденной сметой.

С учетом вышеизложенного, считаем, что анализ выполнения сметы автономного учреждения должен охватывать: оценку выполнения показателей поступлений доходов по отдельным источникам, плана расходов по направлениям финансирования и отраслям производственной и непроизводственной сферы; изучение динамики долговых бюджетных обязательств, кассового исполнения бюджета и стадии его выполнения.

Анализ сметы целесообразно проводить поэтапно. Схематично этапы проведения анализа показаны на рисунке 1.

В тоже время необходимо обратить внимание на тот факт, что в имеющихся научных публикациях отсутствует единый подход к формализации методов и инструментов анализа выполнения сметы. Различные авторы предлагают разнообразные алгоритмы анализа, что свидетельствует о том, что на сегодняшний день не создана унифицированная модель, или дорожная карта аналитика. Это еще раз подтверждает гипотезу о недостаточности применения аналитических приемов в учетной и контрольной работе.

На следующем этапе исследования рассмотрим более подробно особенности проведения анализа выполнения сметы в части выделения автономному учреждению бюджетных ассигнований.

В ходе проведения анализа выполнения сметы по бюджетным ассигнованиям традиционно используется один из главных приемов анализа - сравнение. Сравнение как способ исследования осуществляется через сопоставление одного показателя с другим для определения общих черт или различий между ними, например, сравнение фактических показателей с показателями предыдущих периодов и с плановыми показателями [3]. Важным этапом анализа исполнения сметы является изучение соотношения между кассовыми и фактическими показателями. Данное соотношение может отражать 


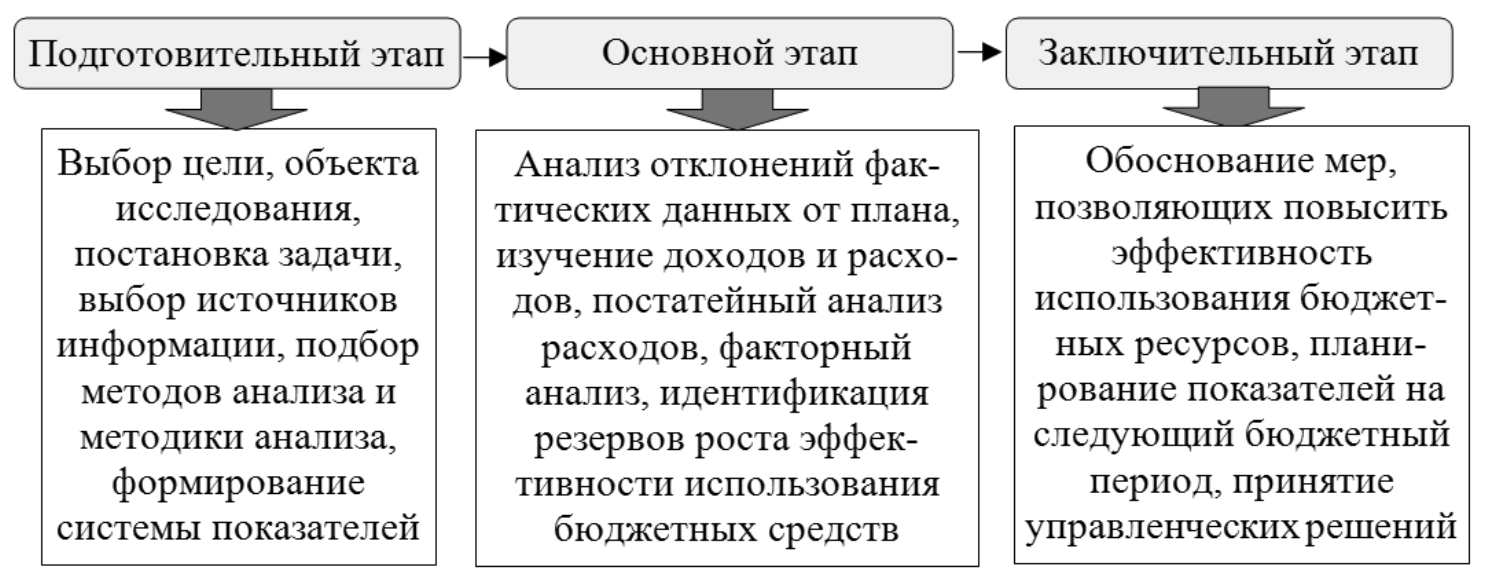

Рис. 1. Этапы анализа исполнения сметы автономного учреждения

превышение кассовых расходов над фактическими или же наоборот - фактических над кассовыми, равенство между ними. Оценка и анализ установившейся между ними зависимости и взаимосвязи позволяет выяснить определенные хозяйственные и финансовые обстоятельства, возникшие в процессе выполнения сметы автономного учреждения.

Осуществляется сравнение расходов по следующим направлениям: фактические расходы и кассовые с расходами, утвержденными сметой; кассовые расходы с фактическими расходами. Поэтому предметом такого анализа являются финансовые ресурсы учреждений, динамика их формирования и использования.

На основании показателей кассовых и фактических расходов, а также показателей доходов фондов учреждения также целесообразно проводить параметрический анализ выполнения сметы, который заключается в определении группы частных и обобщенных показателей, характеризующих все важные элементы сметы [4].

Более подробно параметрический анализ может проводиться по следующим коэффициентам:

- отношение плановых поступлений к фактическим поступлениям средств за отчетный период;

- отношение расходов к доходам;

- отношение расходов внебюджетных средств к сумме собственного капитала.

Рассмотрим некоторые практические случаи отклонений между финансовыми показателями и ситуации, о которых они могут свидетельствовать.

1. Превышение фактических расходов над утвержденными ассигнованиями. Это может свидетельствовать о:

- непринятии руководством автономного учреждения необходимых мер по приведению расходов в соответствие с бюджетными ассигнованиями; взятие обязательств, превышающих ассигнова- ния, что является бюджетным правонарушением и приводит к образованию не бюджетной задолженности;

- получении работ, услуг, товаров, которые на начало года находились в составе дебиторской задолженности.

2. Фактические расходы меньше утвержденных ассигнований, что может сигнализировать о:

- экономичном и рациональном использовании бюджетных средств;

- планирования лишних ассигнований;

- образовании дебиторской задолженности, накоплении запасов;

- несвоевременном отражении в бухгалтерском учете операций, связанных с фактическими расходами.

3. Превышение фактических расходов над кассовыми свидетельствует о:

- погашении дебиторской задолженности, учтенной на начало года;

- образовании кредиторской задолженности на конец отчетного периода;

- списании запасов, приобретенных ранее.

4. Кассовые расходы меньше утвержденных ассигнований. Данная ситуация является индикатором:

- уменьшения ассигнований;

- неполного финансирования учреждения.

Таким образом, подводя итоги проведенного исследования, можно сделать следующие выводы.

Повышение эффективности деятельности автономных учреждений в большинстве зависит от обоснованности, своевременности и целесообразности принимаемых решений. Все это может быть достигнуто в процессе анализа выполнения сметы. Однако только правильно организованная работа по аналитическому исследованию результатов деятельности учреждения может обеспечить его действенность и эффективность, обоснованно повлиять на общий ход процесса функционирования 
учреждения и эффективность выполнения им бюджетных планов и программ.

Представляется, что для повышения качества аналитической информации и обеспечения автоматизации аналитических работ с целью сокращения затрат на их проведение целесообразно внедрить в деятельность автономных учреждений качественное унифицированное программное обеспечение, которое будет адаптировано к актуальным требованиям законодательства, а также оснащено понятным и доступным различным пользователям интерфейсом.

\section{ЛИТЕРАТУРА}

1. Лазарова Л.Б., Бугулов В.В. Пути повышения эффективности расходов бюджетных средств // Гуманитарные и социально-экономические науки. 2018. №6(103). С. 117-120.

2. Чулков А.С. Повышение качества государственных и муниципальных услуг и пути оптимизации сети учреждений, оказывающих их // Региональная экономика: теория и практика. 2020. Т. 18. №1(472). С. 67-83.

3. Мороз Ю.С. Проблемы субсидирования бюджетных и автономных учреждений // Экономика и социум. 2018. №11(54). С. 676-679.

4. Cheng, Yuan; Yang, Lang Providing Public Services Without Relying Heavily on Government Funding: How Do Nonprofits Respond to Government Budget Cuts? // American review of public administration. 2019. Volume 49: Issue 6; pp 675-688.

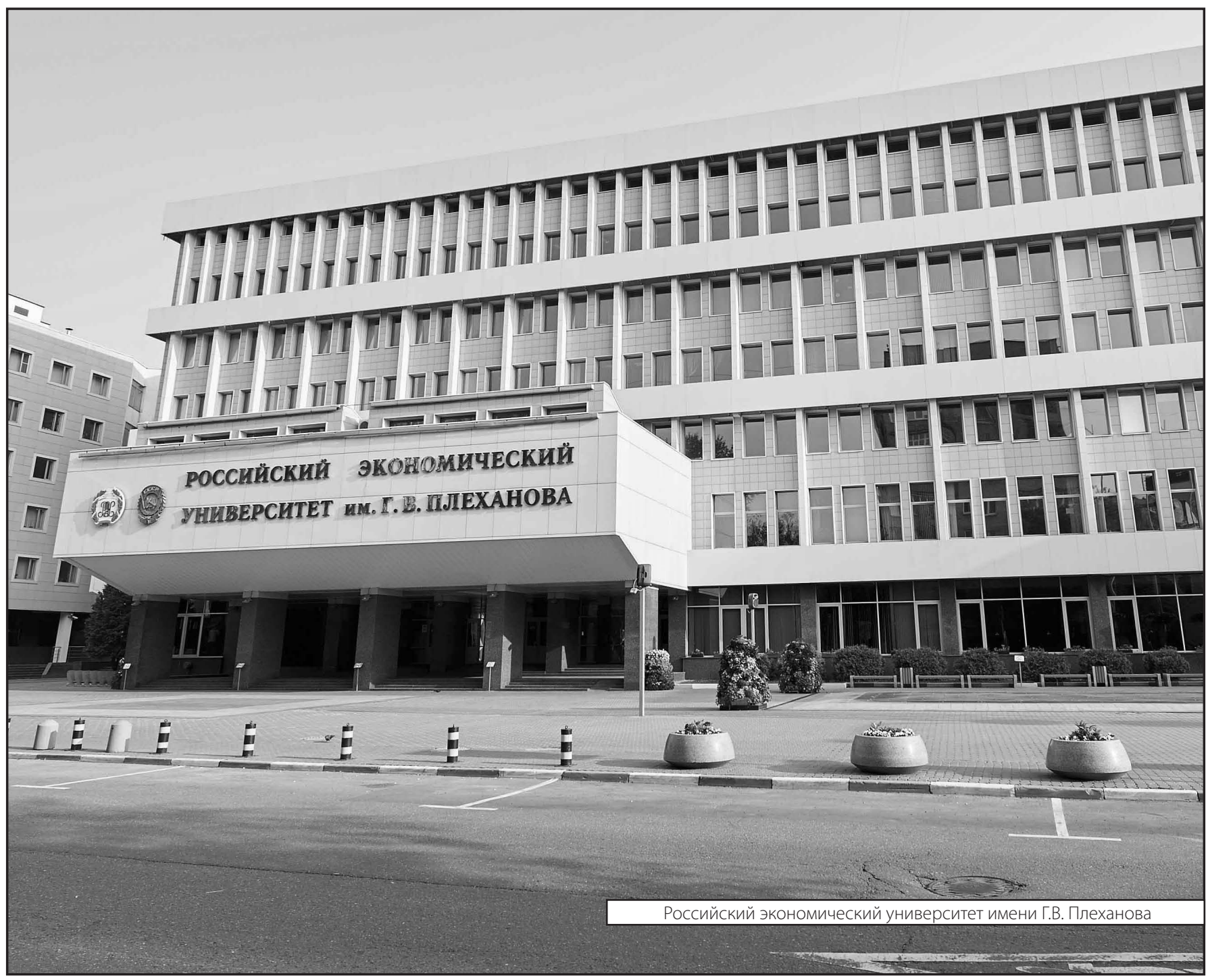

